\title{
ARTE Y BELLEZA EN LA ESTÉTICA DE SCHELLING. LA INDIFERENCIA DE LO IDEAL Y REAL EN PHILOSOPHIE DER KUNST
}

\author{
MAGDALENA BOSCH \\ Universitat Internacional de Catalunya
}

\begin{abstract}
RESUMEN: Este artículo analiza la idea de arte como indiferencia entre lo ideal y real en la Filosofía del Arte de Schelling. Se trata de un momento culminante de la obra de este autor, en la que concentra conceptos esenciales tratados en otros textos como el Sistema, la Filosofía de la naturaleza o Del yo como principio de la Filosofía. Este análisis descubre también otras oposiciones primordiales: entre infinito y finito, la libertad y la naturaleza, lo consciente y lo inconsciente. Estos polos opuestos se relacionan según la ontología del Absoluto que se desarrolla a lo largo de las obras mencionadas y se recoge, más maduramente, en el texto de 1802-3. Este estudio detalla la contradicción ideal-real, su superación a través de la obra de arte, la relación de verdad y belleza como representación del absoluto, cómo belleza y arte realizan ese absoluto, y la dinámica del espíritu ideal penetrando la naturaleza real.
\end{abstract}

PALABRAS CLAVE: Estética de Schelling; Schelling; arte; belleza; Philosophie der Kunst.

\section{Art and Beauty in Schelling's Aesthetics. The Indifference of the Ideal and the Real in Philosophie der Kunst}

ABTRACT: This article analyzes the idea of art as indifference between the ideal and the real within Philosophy of Art, by Schelling. It is a culminating moment for the work of this author. He concentrates essential concepts already explained in other works like System, Philosophy of nature, Of the I as a principle of Philosophy in it. This analysis discover also other primordial oppositions: between finite and infinite, freedom and nature, the conscious and the unconscious. This opposed terms are each other related within the context of the Ontology of the Absolute, that Schelling described in the mentioned works and it is recollected a more robust version in the 1802-3 text. The present study considers the ideal-real contradiction, its overcoming through the work of art, the relationship of truth and beauty as a representation of the absolute, how art and beauty fulfill this absolute, and the dynamics of the idea spirit penetrating the real nature.

KEY WORDS: Schelling's Aesthetics; Schelling; art; beauty; Philosophie der Kunst.

\section{La CONTRADICCIÓN IDEAL-REAL}

Filosofía del arte (1802-1803) es una obra cercana a la producción del primer periodo del pensamiento de Schelling (1795-1801), marcado por la Filosofía de la Naturaleza, la Ontología del Absoluto y la Filosofía de la Identidad ${ }^{1}$, época esta donde se suelen enmarcar estas lecciones. Lo que se considera su Filosofía

1 Sobre estos contenidos clave de la filosofía temprana de Schelling véase: MEIER, F., Die Idee der Transzendentalphilosophie beim jungen Schelling, Keller, Winterthur 1961. 
temprana dialoga comprometidamente con $\mathrm{Kant}^{2}$, Fichte y Spinoza ${ }^{3}$. Además de mantener fuertes resonancias platónicas ${ }^{4}$ y una profunda afinidad con Leibinz ${ }^{5}$. También se nutre del pensamiento de grandes filósofos y contribuye a la formación de un contexto cultural nuevo que se manifestará en la obra romántica de autores como Hölderlin, Novalis o Schiller ${ }^{6}$.

El tema de la obra que tratamos es la relación de la filosofía con el arte ${ }^{7}$. De esta relación entre la razón y lo artístico surge algo nuevo: la posibilidad de acceder a la Indiferencia ideal-real, que no era accesible a la razón. El arte viene a ser un reflejo de la Filosofía, por esto «la Filosofía puede mostrar su ser interno en la Filosofía del arte como en un espejo mágico y simbólico» ${ }^{8}$. De hecho la Filosofía del arte sería "como un círculo más estrecho dentro de la Filosofía, en el que contemplamos directamente lo eterno ${ }^{9}$. La conclusión de este discurso acabará señalando que sólo la razón comprende la unidad primordial, aunque sólo el arte consigue la

2 Resulta interesante la explicación de STAмвоvsкy sobre algunos aspectos de los antecedentes kantianos de la filosofía de la ciencia de Schelling, base fundamental de su Filosofía del arte: SтAмвоvsкy, P., «Idealism, scientia intuitiva, and scientific philosophy», The Review of Metaphysics 66 (December 2012): 339-352, pp. 339-343. Finchman ha elaborado un estudio sobre la recepción de la filosofía trascendental kantiana y la monadología de Leibniz por parte de Schelling y su evolución a lo largo de su obra: Finchman, R. M., "Reconciling Leibnizian monadology and kantian criticism», British Journal for the History of Philosophy, 2015, Vol. 23, No. 6, 1033-1055, http://dx.doi.org/10.1080/09608788.2015.11 05781.

3 La presencia de Kant, Fichte y Spinoza en la obra primera de Schelling está fuera de discusión. Las alusiones a ellos son muy claras y todos los autores lo reconocen así. Por ejemplo, este es el modo en que lo resume Blaumer: «La filosofía temprana de Schelling puede entenderse como una modificación de la Filosofía Crítica de Kant bajo la consideración de los Filosofemas de Fichte y Spinoza». Blaumer, M., Subjektivität und ihr Platz in der Natur, Untersuchung zu Schelling Versuch einer naturphilosphischen Grundlegung des Bewusstseins, Kohlhammer, Stuttgart 2006, p. 35 Además de la fuerte influencia platónica que mencionaremos a parte, y la de Leibniz, señalada — entre otros- por N. Hartmann.

${ }_{4}$ Lo tratamos brevemente a continuación. Uno de los textos más relevantes al respecto es el de Beierwaltes, W., "El neoplatonismo de Schelling», Anuario Filosófico, 33, 2000, pp. $395-442$.

5 Hartmann, N., Die Philosophie des Deutschen Idealismus, Walter de Gruyter, Berlin, New York 1974, p. 113. Destacan también otros estudios analizando la presencia de Leibniz en el pensamiento de Schelling. Concretamente sobre la teoría preestablecida, puede verse también: Braun, O., Schellings geistige Wndlungen in den Jahren 1800-1810, Quelle und Meyer, Leipzig 1906, pp. 15 y ss.

6 Para una revisión bibliográfica de los antecedentes y también del impacto en el Romanticismo literario ver: THIELKE, P., «Recent work on early German Idealism 1781-1801», Journal of the History of Philosophy, vol. 51, no. 2 (2013) 149-192.

7 Para una introducción general a la Filosofía del arte de Schelling véase: PAREYSON, L., Estetica dell'idealismo tedesco, (vol.III: Goethe e Schelling), Mursia, Milano 2003, pp. 295-307.

8 Knittermeyer, H., Schelling und die Romantische Schule, Reinhardt, München 1947, p. 334 (Trad. mía)

9 Cfr. Filosofía del arte, SW 364. Sämtliche Werke (SW). En adelante los textos de Filosofia del arte se citarán, como es usual, con las iniciales SW seguidas del número de página. La numeración que tomamos de referencia corresponde a la edición original del hijo de Schelling, también reproducida en la edición de Schröter y en edición española de López Dominguez. 
mencionada indiferencia de lo ideal y real. El filósofo, con la razón, puede conocer la idea en sí; pero el artista conoce su reflejo en la realidad ${ }^{10}$.

En la Filosofía del arte Schelling aplica esquemas que forjó en el Sistema del idealismo transcendental (1800) y recupera otros que planteó en su Filosofía de la Naturaleza ${ }^{11}$. La presencia de su doctrina física sobre el alma del mundo y la derivación a partir de un todo, tiene raíces en el Systemprogramm ${ }^{12}$ y profundas deudas con Plotino ${ }^{13}$. El Absoluto del que habla Schelling en 1802 no es diverso al que analizaba en textos anteriores. La continuidad con las bases que asentó en el Sistema se refleja tanto en el tema como en el modo de plantearlo. Ya en el Sistema se resolvía una cuestión estética: sólo la belleza resuelve la contradicción finitoinfinito. Ella es causa y resultado de tal superación ${ }^{14}$. Pero además, la imaginación estética proporciona la única mirada capaz de intuir el Absoluto: «la mirada intelectual como imaginación estética proporciona el acto original de una mirada sin intermediarios» ${ }^{15}$.

Esa contradicción de finito-infinito, resuelta en el Sistema, encuentra su paralelo en la Filosofía del arte bajo la forma del binomio ideal-real. Así como la belleza es la superación de la contradicción finito-infinito; así mismo el arte es la superación de la contradicción idea-real: «En la filosofía del arte se une algo contradictorio: el arte o bien lo real, y la Filosofía o lo ideal» ${ }^{16}$. Toda la Filosofía del arte se apoyará en esta relación, que es su punto de partida y lo que le otorga su misión: «se podría determinar de antemano la misión de la filosofía del arte así: representar en lo ideal lo real que está en el arte. Sólo que la cuestión es precisamente qué quiere decir: representar algo real en lo ideal y, mientras no lo sepamos no tenemos claro el

10 Cfr. López Domínguez, V., «Estudio introductorio», Filosofía del arte, Tecnos, Madrid 1999, p. XXI.

${ }_{11}$ Si bien esta coherencia con su filosofía temprana es generalmente reconocida, y la iremos tratando a lo largo del artículo; ha pasado más desapercibida la deuda de todo el Romanticismo y de Schelling en concreto respecto del concepto Bildung propio de la mística medieval alemana. Lo ha estudiado Rius en su artículo: Rius, C., «La funció de l'artista en el pensament romàntic alemany», Comprendre, Vol. 13/2 Any 2011, pp. 105-131.

12 Beierwaltes, W., «El neoplatonismo de Schelling», Anuario Filosófico, 33, 2000, pp. 395-442, p. 410.

$13 \quad$ Ibid., pp. 413-18.

14 «Que todo el sistema cae entre dos extremos, uno de los cuales es designado por la intuición intelectual, el otro, por la intuición estética. Lo que la intuición intelectual es para el filósofo lo es la estética para su objeto. (...) La filosofía alcanza ciertamente lo supremo, pero lleva hasta este punto, por así decir, sólo a un fragmento del hombre. El arte lleva a todo el hombre, como él es, allí, a saber, al conocimiento de lo supremo, y en esto se basa la eterna diferencia y el milagro del arte». F. W. J. Schelling, (1800) System des transzendentalen Idealismus, (SW 1) Esta numeración de las páginas del Sistema corresponde a la edición de K. F. A. Schelling, SÄMTLICHE Werke (SW), J. G. Cotta, Stuttgart, 1856-1861. La traducción que cito es de J. Rivera de Rosales y V. López Dominguez, Anthropos, Barcelona 1988. Para una valoración estética del Sistema véase: Bosch, M.; «Significado estético del Sistema de Idealismo Trascendental», Contrastes. Revista Internacional de Filosofía, vol. XX-N², 2016, pp. 5-25.

15 Blanchard, G.; Die Vernunft und das irrationale. Die grundlage von Schellings Spätphilosophie im «System des transzendentalen Idealismus» und der Identitätsphilosophie, Haah Herchen, Frankfurt Main 1979, p. 49.

16 Cfr. Filosofía del arte, Sämtliche Werke, SW 364. 
concepto de la filosofía del arte» ${ }^{17}$.

También vemos continuidad entre el Sistema y la Filosofía del arte en la correspondencia que establece entre las tres potencias del mundo real e ideal y las tres ideas de verdad, bondad y belleza en el organismo y en el arte. Las tres potencias son resultado del autoponerse del yo: la primera es lo primero pensable, lo que es en sí; pero no objetivamente, porque todo lo objetivo pone algo fuera de sí; sino en el sentido de lo que es, que es en sí. La segunda lo que es sólo un ser objetivo sin subjetividad, y la tercera: nada puede pensarse sólo como sujeto; nada puede ser objeto si no es frente a un sujeto; y nada puede permanecer siendo ambos ${ }^{18} \cdot$ ¿Cómo se da la correspondencia entre potencias y las tres ideas mencionadas? "A la primera potencia del mundo ideal y real corresponde la verdad, a la segunda el bien, a la tercera la belleza» ${ }^{19}$. De este modo vemos ya que la condición del concepto de belleza en la filosofía del arte es realizar la tercera potencia, en la que se da la superación del enfrentamiento sujeto-objeto.

Otro concepto especialmente cercano al Sistema, es el de lo orgánico: lo organizado interiormente, lo que se define a sí mismo desde dentro, es parte importante de aquello real y vivo de que se trata con frecuencia en ese texto. Respecto a lo orgánico el arte viene a ser su grado máximo, es decir, su culminación. El arte es lo orgánico en su forma más altamente desarrollada. En el arte esa disposición interior que admiramos en la naturaleza, incluso en algunas naturalezas simples, se realiza de modo igualmente interno y vivo pero en su máxima perfección y complejidad creando "productos organizados superiores ${ }^{20}$.

\section{LA INDIFERENCIA DE LO IDEAL Y REAL EN EL ARTE}

En Filosofía del arte vemos cómo la comprensión de la disciplina que trata, determina el papel de la belleza en esta obra y en esta ciencia. Desde el inicio se remite o se presupone la definición que se dio en el Sistema según la cual, belleza es lo infinito en lo finito. Pero no se acaba toda esta nueva obra de Schelling con una mera repetición de lo que ya se había definido; sino que manteniendo esta definición se ofrecen otras nuevas, perfectamente coherentes con ella, pero que la amplían y la aclaran: «la producción artística realiza la unión de lo ideal y real del yo, superando la general contradicción de producción y reflexión, tal como le corresponde; y así puede cumplir el postulado de la intuición estética. Así terminan ambos en la unión y la identidad ${ }^{21}$.

Esta explicación cuenta con una aclaración complementaria, breve pero muy directa, en la lección XIV de las Lecciones sobre el método de los estudios académicos. Allí se afirma que la relación entre la filosofía y el arte viene a ser la repetición, en su última potencia, de la relación entre lo real y lo ideal, que se mantiene allí

17 Ibid., 364.

18 Cfr. JAspers, K., Schelling, Grösse und Verhängnis, Piper, München 1986, pp. 132-133.

19 Filosofía del arte, SW 382.

$20 \quad$ Ibid. SW 358.

21 Barth, B., Schellings Philosophie der Kunst, Gottliche Imagination und ästhetische Einbildungskraft, (Symposion Bd. 92), Alber, München 1991, p. 132. 
donde toda oposición desaparece en la absolutidad, en los confines de lo finito y lo infinito 22 .

Dos de los grandes principios que se establecen a lo largo de la Filosofía del Arte, se exponen en las Lecciones de modo claro y directo: el único propósito del arte es la producción de la belleza, y que esta belleza es algo muy distinto del placer de las puras excitaciones de los sentidos. Éste es un error muy frecuente que el filósofo advertirá sin dificultad, porque su objeto de estudio propio es la verdad y, si se la conoce, se descubre el engaño de un modo artístico en el que el papel de la belleza es usurpado por la mera sensualidad ${ }^{23}$.

Desde esta nueva intuición estética puede el filósofo explicar la naturaleza del verdadero arte, de lo que debe ser o de cómo hemos de entenderlo. En cualquier caso, Schelling expone su idea de arte y por tanto, el concepto que rige la Filosofía del arte:

«Pero yo me refiero a un arte más sagrado, a ese arte que, según expresiones de los antiguos, es un instrumento de los dioses, revelador de misterios divinos, manifestación de las ideas, de la belleza ingénita, cuyo rayo inmaculado sólo ilumina interiormente las almas puras y cuya figura permanece tan oculta e inaccesible a los ojos sensibles como la de la verdad misma» ${ }^{24}$.

La ciencia que se nos propone es una ciencia sublime, que se ocupa del más alto objeto de contemplación. La Filosofía del arte está condicionada por una consideración de la belleza como ingénita, muy cercana a la idea platónica. Pero si Schelling comparte con Platón una idea del arte tan elevada, unida a una idea de belleza divina, ¿Cómo no entrar en conflicto con su valoración de los artistas? pues según la perspectiva platónica ellos son perniciosos engañadores ${ }^{25}$. La explicación que da Schelling es que Platón se refiere al arte que no cumple, precisamente, los requisitos que él mismo exige para considerarlo verdadero arte. Y también se trata de una cuestión circunstancial: Platón conoció artistas que no fueron suficientemente espirituales; pero actualmente contamos con el ejemplo de «la poesía cristiana que en su conjunto lleva impreso el carácter de lo infinito de un modo tan definido como la poesía antigua en general lleva el de lo finito» ${ }^{26}$.

El filósofo tiene la misión estrechamente relacionada con la de los artistas: reconocer y representar en el arte una verdad superior ${ }^{27}$. Esta misión es la que se va realizando o cumpliendo a lo largo de la filosofía del arte. El arte mismo aporta ya una potencia más alta que la potencia de la naturaleza ${ }^{28}$. Hasta el momento, considera Schelling que esa tarea no se ha conseguido aún, está en un estado, podríamos decir, embrionario: «sólo existen unas semillas» ${ }^{29}$ de la ciencia que ha de ser la filosofía del arte, una auténtica ciencia del arte que tome en cuenta la verdad sobre la belleza y

${ }^{22}$ Cfr. Schelling, F. W. J., Lecciones sobre el método de los estudios académicos. Lección XIV, SW 348. (Paginación correspondiente a la edición del hijo de Schelling).

${ }^{23}$ Cfr. Lecciones, SW 345.

24 Cfr. Ibid., SW 345.

25 Cfr. Platón, República, 597e-598d.

26 Lecciones, SW 347.

27 Ibid., SW 350.

${ }_{28}$ Knittermeyer, H., Schelling und die Romantische Schule, Reinhardt, München 1947, p. 343.

29 Cfr. Lecciones, SW 351. 
haciendo justicia a lo que ella es, desarrolle un todo científico del arte que la busca solo a ella y la reproduce. Ambas afirmaciones sobre la ciencia del arte, a saber, que es de suma importancia y que no ha sido desarrollada todavía; se repiten en las Lecciones sobre el método de los estudios académicos y al inicio de la Filosofía del arte.

Los juicios sobre el arte son cambiantes, en ellos predomina a veces el criterio de la verdad, otras el de la belleza; pero en ningún caso saben los que juzgan qué es verdad ni qué es belleza. Y este desacuerdo es general, entre los que practican el arte ${ }^{30}$. Acerca de las posibilidades de una ciencia del arte, se nota la carencia de unos principios sobre los que poder basar esa ciencia, pues no se han establecido aún unos principios absolutos. A pesar de la Crítica del Juicio de Kant y de algunas aportaciones de los kantianos, ninguno ha construido aún «...un todo científico, o por lo menos, establecido los principios absolutos con validez universal y forma rigurosa; en la mayoría no se produjo la separación estricta del empirismo y la Filosofía, exigida por la verdadera cientificidad ${ }^{31}$. Este es precisamente el propósito de Schelling en esta Filosofía del arte: edificar esa ciencia del arte que está por fundar.

\section{VERDAD y BELLEZA COMO REPRESENTACIÓN DEL ABSOLUTO}

Para fundar esta nueva ciencia parte de la comprensión de la obra de arte como producción que presenta a nuestra conciencia la infinitud que brota de nuestro inconsciente $^{32}$. Por eso también esta ciencia del arte se debate entre los dos polos de la contradicción antes enunciada. «El arte es lo real, lo objetivo; la filosofía lo ideal, lo subjetivo» ${ }^{33}$. Pero como ya se ha expuesto alguna vez, la contradicción se resuelve en la absolutidad; y entre la filosofía y el arte también será lo absoluto lo que permita una unidad. No en vano el absoluto incondicionado es el centro del Sistema ${ }^{34}$. Porque toda la Filosofía del arte será en realidad una continuidad de la Filosofía de la Naturaleza, en el sentido de que sigue siendo una Filosofía de la Unidad ${ }^{35}$.

Y esta unidad, como se ha apuntado más arriba y también de manera parecida a como ocurría en Bruno, se cumple en el absoluto. Al final del diálogo se concluye la identidad de verdad y belleza. También en la Filosofía del arte la relación de verdad y belleza es un aspecto esencial. Para Schelling verdad y belleza son «equiparable representación de lo Absoluto, que se hace evidente en los diversos medios del Arte y de la Filosofía ${ }^{36}$. Pero además, y por esta misma razón, verdad y belleza se necesitan mutuamente, vemos que «La verdad sin belleza se reduce a una verdad relativa y la belleza sin verdad se rebaja a la habilidad de la ejecución y se limita al aspecto sensible

\footnotetext{
30 Filosofía del arte, SW 361.

31 Ibíd., 362.

32 Cfr. Kogan, J., Arte y metafísica, Paidós, Buenos Aires 1971, p. 60.

33 Filosofía del arte, SW 364.

34 Cfr. SKariKa, M., «Determinismo o libertad. La idea de libertad en Schelling», Anuario
} Filosófico, (27) 1994, pp. 719-730. p. 723.

35 Hartmann, N., Die Philosophie des Deutschen Idealismus, Walter de Gruyter, Berlin, New York 1974, p. 115.

36 Barth, B., Schellings Philosophie der Kunst, Gottliche Imagination und ästhetische Einbildungskraft, (Symposion Bd. 92), Alber, München 1991, p. 43. 
opacando el resplandor de la naturaleza eterna ${ }^{37}$. La relación entre filosofía y arte se basa en la relación entre verdad y belleza, en el modo en que ambas son representaciones del absoluto. Por eso el arte se basa en esa identidad y la belleza se identifica con la verdad, pues tanto verdad como belleza consisten en cierta identidad de lo subjetivo y lo objetivo: «la verdad como modelo, la belleza como imagen reflejada» ${ }^{38}$.

La unidad de verdad, bien y belleza se afirma como condición necesaria para las tres ideas:

«La verdad que no es belleza tampoco es verdad absoluta, y a la inversa. (La oposición de verdad y belleza, tan frecuente en el arte, se basa en que se entiende por verdad exclusivamente la verdad falaz, la que sólo alcanza lo finito). De la imitación de esta verdad resultan esas obras de arte en las que sólo admiramos el artificio con que se consiguió en ellas lo natural sin unirlo a lo divino. Esta clase de verdad, sin embargo, no es aún la belleza en el arte. Sólo la belleza absoluta en el arte es también la auténtica y legítima verdad.

Por la misma razón, el bien que no es belleza tampoco es bien absoluto, y a la inversa; pues en su absolutidad el bien se vuelve también belleza, por ejemplo, en todo espíritu cuya moralidad no se funda en la lucha de la libertad con la necesidad sino que expresa la armonía y la conciliación absolutas» ${ }^{39}$.

Aquí se repite de modo casi idéntico aquella argumentación que se lee en Bruno acerca de la identidad entre verdad y belleza: que se identifican en lo absoluto, y que ni la verdad ni la belleza son verdaderamente tales, ni pueden ser perfectas, si no son a la vez bella y verdadera respectivamente. De estas condiciones se derivan al menos dos consecuencias: la unitaria concepción del universo, y las condiciones de esa unidad. La unidad del universo y con ella, la posibilidad de que sea reconocida; se realiza en el organismo. «La síntesis de real e ideal que significa el organismo es la perfecta revelación de lo divino, pero nunca de las dos dimensiones por separado ${ }^{40}$.

Hay otra dimensión de la unidad en la que se funden los dos universos ideal y real. Ese universo que es uno y que comprende ambos en completa unidad, es el universo que "está formado en Dios como obra de arte absoluta y en eterna belleza ${ }^{41}$. Sin embargo - y esta es la segunda consecuencia - la unidad que se da en la perfección, esa unión entre verdad y belleza, sólo puede ser observada por el espíritu que tenga esa capacidad: sólo un espíritu armónico «siente de veras la poesía y el arte», que no pueden enseñarse sino que han de ser intuidos por un espíritu capaz. "Los arquetipos de todas las cosas son absolutamente verdaderos y absolutamente bellos. En cambio, lo perverso, lo feo, igual que el error y lo falso, consisten en una mera privación y sólo tienen que ver con la contemplación temporal de las $\operatorname{cosas} »^{42}$. La contemplación temporal de las cosas, en cambio, es parcial y defectuosa. Es decir, según se contempla, se ve o no lo perfecto o lo

37 López Domínguez, V., «Estudio introductorio», Filosofía del arte, Tecnos, Madrid 1999, p. XXI.

38 Cfr. Filosofía del arte, SW SW 384.

39 Ibid., 385.

40 De Torres, M. J., «Estética y religión en la filosofía del arte de Schelling», en Leyte, A.

(Ed.) Una mirada a la filosofía de Schelling, Universidad de Vigo, Vigo 1999, p. 123.

$41 \quad$ Filosofía del arte, SW 385.

42 Ibid., SW 386. 
absoluto. Pues existe un único ser absolutamente real ${ }^{43}$. «En la filosofía no hay más que lo absoluto» ${ }^{44}$ y la verdadera obra de arte es un todo bello, un cierto absoluto.

De lo absoluto único se desprende todo conocimiento y toda ciencia, por tanto también la filosofía y el arte. De modo que hay una correspondencia o simetría entre verdad y belleza de manera paralela a como la hay entre filosofía y arte. En efecto, tanto el arte como la Filosofía se refieren a arquetipos, aunque difiere el arquetipo concreto al que se refiere cada una: «Así como para la filosofía lo absoluto es el arquetipo de la verdad, para el arte es el arquetipo de la belleza», de esta singular relación se deriva también que la «filosofía intuye las ideas como son en sí, el arte las intuye realmente ${ }^{45}$. Es decir, la unidad o identificación en lo absoluto, una análoga vinculación con los arquetipos; y una diferencia decisiva: la filosofía no trata de las cosas reales, el arte sí.

En ambos casos, filosofía y arte, la unidad del objeto de estudio responde a las exigencias de la disciplina de que se trata. Unidad de la filosofía: hay una única filosofía. Si se habla de distintas ciencias de la filosofía «o bien es algo del todo errado, o sólo son representaciones del todo único e indiviso de la filosofía en diferentes potencias o bajo determinaciones ideales distintas ${ }^{46}$. Y en el arte ocurre lo mismo: sólo el todo es bello en el arte, sólo en la Indiferencia del todo se da la belleza. La unidad del arte no se refiere sólo a la ciencia; sino también a sus productos: formando una unidad con el universo y sus predeterminaciones, surgidos todos de un mismo genio ${ }^{47}$.

La relación real entre filosofía y filosofía del arte es claramente la de una parte dentro del todo: es decir, la filosofía del arte es una parte de la filosofía, pero a la que corresponde la contemplación de la belleza: «En la filosofía general disfrutamos viendo el severo rostro de la verdad en sí y por sí misma; en esta esfera particular de la filosofía que circunscribe la filosofía del arte alcanzamos la intuición de la belleza eterna y los arquetipos de todo lo bello» ${ }^{48}$. Pero nada más lejos de la filosofía del arte que una idea de parcialidad. Todo objeto de la filosofía, para poder serlo, ha de «estar él mismo fundado en lo absoluto por una idea eterna y necesaria ${ }^{49}$. Por eso, en la filosofía del arte «construyo el universo en la figura del arte» y aún más, explícitamente se afirma en más de una ocasión el carácter total de la filosofía del arte: «en la filosofía del arte, no construyo el arte como particular, sino que la figura del arte es la ciencia del todo en la forma o potencia del arte ${ }^{50}$. De nuevo Filosofía y Arte nos remiten al absoluto.

\section{LA CIENCIA DEL ARTE Y LA ONTOLOGÍA DE LO ABSOLUto}

Algunas veces lo absoluto es Dios. Pero esta identificación no es estática, permanente y recíproca. Dios es solamente un aspecto de lo absoluto o el todo,

\footnotetext{
43 Ibíd., SW 366.

44 Ibid., SW 367.

45 Ibid., SW 370.

46 Ibid.,SW 365.

47 Cfr. Ibíd., SW 372.

$48 \quad$ Ibid., SW 364.

$49 \quad$ Ibid., SW 367.

${ }^{50} \quad$ Ibid., SW 368.
} 
que comprende otros momentos o realidades. "Mundo y Dios no son sino la cara real y la ideal de una sola cosa; y en el todo está contenido lo que en Dios está comprendido ${ }^{51}$. Pero al tratar de la construcción del arte, más de una vez se equiparan Dios y lo Absoluto como si se tratara de una misma realidad: «Lo absoluto o Dios es aquello respecto de lo cual el ser o la realidad se deriva inmediatamente a partir de la idea gracias a la mera ley de identidad, o sea, Dios es la afirmación inmediata de sí mismo» ${ }^{52}$.

El contexto de esta afirmación es la «construcción del arte», título de la primera sección de la Filosofía del arte, donde desde el inicio se señala esa identidad de lo absoluto y Dios. Y es que esa identidad condiciona el arte, porque sólo es verdadero arte el que hace referencia a lo divino, que es la interioridad y la verdad de lo natural. Indica Galán: «Por eso se queja Schelling contra los que toman la forma externa como referencia de sus trabajos artísticos, porque dejan abierto un vacío que no puede ser llenado, allí donde se espera encontrar lo perfecto, lo verdadero y la belleza suprema ${ }^{53}$. En esto se parece el Absoluto y lo divino, en que para el arte ambos están en ese otro lugar que es lo infinito y que sólo a través de la belleza se hará presente en lo finito.

Este es el motivo de que una obra que trata de la ciencia del arte, es más; que aspira a construir y fundamentar por primera vez una verdadera ciencia del arte, empiece haciendo referencia al Absoluto. Si construir el arte es determinar su lugar en el universo, se ha de atender a lo Absoluto, en referencia al cual se sitúa cualquier parte del universo. Y eso remite a la idea de Dios: «Dios es el universo absoluto inmediatamente en virtud de su idea. De la idea de Dios se sigue de inmediato lo infinito y se sigue necesariamente de manera infinita, pues, en cuanto afirmación infinita de sí mismo, Dios también se comprende a su vez infinitamente como afirmante, infinitamente como afirmado, e infinitamente como indiferencia de ambos» ${ }^{54}$. Lo absoluto es identidad, totalidad, eternidad; pero ni consciente ni inconsciente, ni libre ni no libre. Se halla fuera de estas últimas categorías, y es precisamente en el arte donde se pone en relación con ellas: la fuerza creadora es libre, la creatividad de la naturaleza es inconsciente.

A medida que se define el absoluto y su identificación con Dios, se anuncia también una identidad entre Dios y el universo. Esta identidad se remonta a los escritos sobre la Naturaleza y a sus deudas con el alma del mundo del Timeo. Para Platón, Dios, siendo el alma del mundo, es «el alma de todo» ${ }^{55}$. Casi al principio se había enunciado esta nueva identidad: "Dios y el universo son lo mismo o sólo distintas caras de lo uno y lo mismo» ${ }^{56}$. Es lógico, pues, que su contenido sea el mismo, que lo que comprende uno esté comprendido también en el otro;

51 De Torres, M. J., «Estética y religión en la filosofía del arte de Schelling», en LEYTE, A. (Ed.) Una mirada a la filosofía de Schelling, o.c., p. 123.

52 Filosofía del arte, SW 373.

53 Galán, I., El Romanticismo: F. W. J. Schelling o el arte divino, Ediciones Endymion, Madrid 1999, p.87.

$54 \quad$ Filosofía del arte, SW 375.

55 Cfr. Schlette, H. R., Weltseele. Geschichte und Hermeneutik, Frankfurt, Verlag J. Knecht, 1993, pp. 35-80. Para un análisis del Platonismo y Plotinismo de Schelling véase la ya mencionada obra de Beierwaltes.

56 Filosofía del arte, SW 366. 
y que la naturaleza sea eterna, puesto que es «el infinito ser afirmado de Dios» ${ }^{57}$. Toda comprensión y consideración del universo y de la naturaleza incluirá esta polémica identificación. Porque, tal como plantea Francesc Pereña: «Qué significa, con exactitud, establecer la relación en Dios entre su "naturaleza" y lo que es "él mismo”? ¿Es un Dios que deviene más "en Dios” o ya no es un absoluto devenir "en” cada concreto devenir, tanto en las cosas como en los humanos, como realización de la articulación del ser básica y original? „ ${ }^{58}$

Pero hay que distinguir entre la naturaleza eterna y lo que como tal comprende en sí; de sus manifestaciones, a la fuerza siempre aisladas y en esa misma medida, nunca revelación perfecta de Dios. La revelación perfecta de Dios sólo se da allí donde las formas aisladas se resuelven en identidad absoluta, y eso ocurre solamente en la razón.

Asentados estos requisitos respecto de lo absoluto se podrá comprender el lugar del arte y de la belleza en el universo. Por medio del arte se presenta en el mundo ideal la indiferencia de lo ideal y lo real $^{59}$, y la belleza está allí donde se encuentran lo ideal y lo real:

«Puede decirse que hay belleza siempre que la luz y la materia, lo ideal y lo real, entran en contacto. La belleza no es sólo lo general ni lo ideal (esto = verdad), tampoco lo meramente real (esto es la acción), por tanto es la plena compenetración o unificación de ambos. Hay belleza ahí donde lo particular (real) es tan adecuado a su concepto que éste, en cuanto infinito, ingresa en lo finito y es intuido in concreto. De esta manera lo real en que se manifiesta el concepto va asemejándose verdaderamente e igualándose al arquetipo, a la idea, donde lo general y lo particular se encuentran en absoluta identidad. Lo racional en cuanto racional se convierte al mismo tiempo en algo que aparece, se hace sensible» ${ }^{60}$.

Este es, probablemente uno de los momentos en que más claramente Schelling presenta la belleza como un elemento conciliador de lo que parece inconciliable. Ya en el Sistema del idealismo transcendental proclamaba la solución de la infinita contradicción irresoluble. Aquí, en términos de la filosofía de la identidad, se presenta la belleza como la posibilidad de unión entre el mundo ideal y real, de un modo que supera el profundo platonismo del que parte. Ese mundo ideal y real: intelectual uno, sensible el otro; refleja, en cierto modo, la visión platónica de las ideas. Y sin embargo el modo en que aquí se anuncia una "absoluta identidad entre lo racional y lo sensible» quedaría muy lejos de la concepción platónica.

57 Ibid., SW 378.

58 Pereña, F., «Freiheit und System. Das Problem der Kompatibilität», Schellings Philosophie der Freiheit. Studien zu den Philosophischen Untersuchungen über das Wesen der menschlichen Freiheit. Ferrer und Pedro (Hrsg.), Ergon Verlag, Würzburg 2012, p. 133.

59 Filosofía del arte, SW 380.

${ }^{60}$ «Die Schönheit, kann man sagen, ist überall gesetz, wo Licht und Materie, Ideales und Reales sich berühren. Die Schönheit ist weder bloss das Algemaine oder Ideale (dies = Wahrheit) noch das bloss Reale (dies im Handeln), also sie ist nur die vollkommene Durchdringung oder Ineinsbildung beider. Schönheit ist da gesetzt wo das Besondere (Reale) seinem Begriff so angemessen ist, das dieser selbst, als Unendliches, eintrit in das Endliche und in concreto angeschaut wird. Hierdurch wird das Reale, in dem er (der Begriff) erscheint, dem Urbild, der Idee wahrhast änlich und gleich, wo eben dieses Allgemeine und Besondere in absoluter Identiät ist. Das Rationale wird als Rationales zugleich ein Ercheinendes, Sinnliches». Ibíd., SW 382. 
Junto a esta unidad de lo inteligible y lo sensible, se presenta la unidad entre verdad, bien y belleza. Y este es un aspecto de la relación de la belleza con Dios, pues Él se encuentra por encima de ellas, y no es ninguna de ellas en particular o en exclusiva, sino la unión de todas. La filosofía, de modo parecido, oscila por encima de las tres ideas tratando de lo común a todas. Sólo su determinación formal marca una preeminencia de la verdad como objeto más propio y directo para la ciencia filosófica; y por eso la filosofía no es sólo ciencia, sino también virtud y arte. «No podría pensarse la filosofía sin arte y sin conocimiento de la belleza» ${ }^{61}$.

\section{LA PENETRACIÓN DEL ESPíRITU IDEAL EN LA NATURALEZA REAL}

El protagonismo de la belleza, como elemento en el que se unen los opuestos, tal como se ha visto anteriormente en esta y en otras obras de Schelling, encuentra una de sus más contundentes explicaciones en los argumentos en torno a la libertad. "A la verdad corresponde la necesidad, al bien la libertad ${ }^{62}$. Pero es en la belleza donde se encuentran ambas. En efecto en perfecta coherencia con la explicación de que la belleza es unificación de lo real y lo ideal, se deberá reconocer que la belleza ha de ser también indiferencia de la libertad y la necesidad intuida en algo real. «Llamamos bella, por ejemplo, una figura en cuyo esbozo la naturaleza parece haber actuado con la máxima libertad y la más sublime sensatez, aunque siempre dentro de las formas y los límites de la más estricta necesidad y regularidad. Bello es un poema en el que la máxima libertad vuelve a captarse a sí misma en la necesidad»63.

La relación entre la libertad y la necesidad es también la relación entre el yo y no-yo ${ }^{64}$. Es el fundamento de toda la dinámica del despliegue del yo que da lugar al Sistema de 1800 y que pone las bases de la Filosofía del arte. Es, también, la relación del yo consigo mismo ${ }^{65}$. Los término de ese enfrentamiento son «algo que aparece como lo finito y un indeterminado yo ${ }^{66}$, es decir, lo determinado y lo indeterminado, lo finito y lo infinito. Pero esa es una oposición a la que corresponde dejar de serlo,

${ }^{61} \quad$ Ibid., SW 383.

62 Ibid., SW 383.

63 «Wir nennen z. B. schön eine Gestalt, in deren Enwurf die Natur mit der grösten Freiheit und der erhabensten Besonnenheit, jedoch immer in den Formen, den Grenzen der strengsten Nothwendigkeit und Gesetzmässigkeit gespielt zu haben scheint. Schön ist ein Gedicht, in welchem die höchste Freiheit sich selbst wieder in der notwendigkeit sasst». Ibíd., SW 383.

${ }_{64}$ La idea de libertad en Filosofía del arte está en estado embrionario en el Sistema de 1800. Pero no es hasta más adelante donde se desarrolla plenamente (Investigaciones sobre la libertad humana y los objetos con ella relacionados, 1809). Para un estudio exhaustivo sobre la libertad en este texto y en la etapa posterior, ver Perez-Borbujo, F., Schelling, el sistema de la libertad, Herder, Barcelona 2003.

${ }_{65}$ Warnek llega a afirmar que es la relación de Schelling consigo mismo, que de modo misterioso ahonda en la máxima socrática del autoconocimiento. Este autor lo ve como el fundamento y la continuidad de toda su obra, de principio a fin; y con una estrecha relación respecto de su intelectualidad personal. Cfr. WARNEK, P.; «Schelling's Second Sailing», Epoché: A Journal for the History of Philosophy, 8 (2):195-214 (2004), p. 199.

66 Leyte, A., «Lo infinito romántico», Suplemento de Contrastes. Revista internacional de filosofía, 19 (2014) pp. 205-222, p. 211. 
o ser superada ${ }^{67}$. Es la oposición que sólo en la belleza consigue conciliar y que la Filosofía del arte nos ayuda a comprender: porque en el arte el yo consciente se encuentra con el yo no consciente y precisamente a través de él, por medio de él, en cierto modo gracias a él; la oposición como tal se desvanece.

Pero además de esa interacción entre espíritu y naturaleza emerge una comprensión enteramente nueva de la naturaleza misma y de lo orgánico. La naturaleza viviente no es ni un conjunto de leyes, ni una copia de la inorgánico, ni un halo vital, ni unas estructuras de la razón, ni una fuerza teleológica. Schelling se centra — distinguiéndose de otros autores - en la relación de los organismos con su entorno ${ }^{68}$.

Esa relación se realiza en la interacción entre lo consciente y lo no consciente. Lo consciente es la libertad, lo no consciente es la naturaleza o el universo. Como dice K. Goudell:

«En este sentido es necesario, a la vez, que la obra de arte refleje el universo y que su observador tenga un alma elevada y sensible que hace posible verlo. Es lo que ocurre con el grano de arena, si somos capaces de apreciarlo como reflejo del universo: si podemos ver el universo en un grano de arena, como insistió William Blake, ¿significa esto que un grano de arena es también bello? Siguiendo el pensamiento de Schelling, yo afirmo que el grano de arena visto como reflejo del universo es bello en su representación, y en eso está todo el empeño: tanto el del impacto de la producción artística, como el alcance del ojo artístico» ${ }^{69}$.

Las condiciones en que los arquetipos se reflejan en el mundo real y el modo como accedemos a ellos, determinan un papel esencial para la belleza: Dios mismo como arquetipo se vuelve belleza en la imagen reflejada y también las ideas de la razón intuidas en esa imagen se hacen belleza ${ }^{70}$. Dios y las ideas se hacen belleza cuando se hacen visibles a los seres humanos. El reflejo de la perfección de Dios es la belleza. La medida humana de lo perfecto, la medida en que los humanos pueden ver o intuir lo perfecto, es belleza sólo en la medida en que es reflejo de lo absoluto, de Dios: «Dios es la causa de todo arte y la fuente de toda belleza» ${ }^{71}$. Y por eso el arte, cuyo arquetipo se dijo ser la belleza, deberá representar las formas de cosas bellas, y eso equivale a decir, que deberá representar las formas de las cosas «como son en Dios o como son en sí, como son en lo absoluto» ${ }^{72}$.

Aquí se desarrolla una teoría neoplatónica de la participación en la que no sólo hay cosas en el mundo que participan de ideas; sino que todo participa de Dios. Y a la vez que neoplatónica es spinoziana. El arte que une lo ideal y lo real; teje y a la vez refleja, la relación del espíritu con la naturaleza. Y así, en este

${ }_{67}$ Cfr. Ibid., p. 211.

68 Cfr. KabeshKin, A., "Schelling on understanding organisms», British Journal for the History of Philosophy, 25:6, 2017, 1180-1201, DOI: 10.1080/09608788.2017.1298989, pp. 1183-88.

${ }_{69}$ "However, if in a grain of sand we can see the universe, as William Blake insisted, does this mean that a grain of sand is also beautiful? Thinking with Schelling, I argue that a grain of sand seen as reflecting the universe is indeed rendered beautiful, and this is the whole endeavor, both the leap of artistic production and the purview of the artistic eye». GoudELI, K., "The potences of beauty: Schelling on the question of Nature and art», Comparative and Continental Philosophy, 4. 2, 2012, Sheffield, pp. 253-263, (Trad. mía), p. 254.

70 Cfr. Filosofía del arte, SW 386.

71 Cfr. Ibíd., SW 386.

72 Cfr. Ibid., SW 387. 
sentido afirma P. Genths: «Como la sustancia de Spinoza, el Absoluto viene a ser como la Representación del espíritu en lo ideal y en la naturaleza como real, por separado» ${ }^{73}$. Ahí donde están las ideas, están por el gobierno de la Potencia de la Natura naturata (...) ellas son las formas concretas del ser» ${ }^{74}$.

La verdadera esencia de las formas particulares está en lo absoluto, donde están separadas a la vez que forman una unidad: "Arte y Filosofía tienen el ser uno de Dios y del universo, que — una vez hecha la diferenciación de sus mediosrepresenta el uno en el arquetipo, refleja el otro en la copia, y se halla a sí mismo en una relación cambiante que se basa en su relación común con el Absoluto» ${ }^{75}$. Esta peculiar relación de las formas particulares con lo absoluto determina la visión del universo ya sea como caos o como suprema belleza, siempre será una absoluta identidad o una absolutidad de la forma; y cada forma en la medida en que tiene su esencialidad en lo absoluto es ella misma un universo y se llama idea. Siguiendo estas consideraciones se llega a la conclusión de que lo particular, unido a lo general, es reflejo de lo divino, son dioses ${ }^{76}$.

La unidad del universo comprende la superación de dos dualidades: sujeto-objeto y naturaleza-espíritu. Volviendo al principio y a los fundamentos: la Filosofía de la ciencia tiene que ser unitaria, y esa unidad exige la unión de sujeto y objeto; como también la unión de forma y contenido, que se determinan absolutamente una a otra $^{77}$. De nuevo resuena el eco del Sistema del idealismo fundamental y de la Filosofía de la naturaleza, donde el universo es manifestación del infinito y vive por la vida de un alma divina. Por ese motivo la pregunta por el objeto del arte no está ni siquiera respondida por la Filosofía del arte y es la Física especulativa la que vuelve una y otra vez sobre ello «para conseguir una intuición fundamental de la Totalidad» ${ }^{78}$.

\section{BiBLIOGRAFÍA}

Barth, B. (1991). Schellings Philosophie der Kunst, Gottliche Imagination und ästhetische Einbildungskraft, (Symposion Bd. 92). München: Alber.

Beierwaltes, W. (2000). «El neoplatonismo de Schelling», Anuario Filosófico, 33, pp. 395-442.

Blanchard, G. (1979). Die Vernunft und das irrationale. Die grundlage von Schellings Spätphilosophie im «System des transzendentalen Idealismus» und der Identitätsphilosophie. Frankfurt Main: Haag Herchen.

Blaumer, M. (2006). Subjektivität und ihr Platz, in der Natur, Untersuchung zu Schelling Versuch einer naturphilosphischen Grundlegung des Bewusstseins. Stuttgart: Kohlhammer.

73 Genths, P., Die Identitäts Philosophie Schellings in ihrem Verhältnis zur Religion, Memminger, Würzburg, 1926, p. 27.

${ }^{74}$ Como mencionamos al principio, también se pone en evidencia la herencia de Spinoza, repetidamente mencionada por varios autores, por ejemplo: GentHs, P., Die Identitäts Philosophie Schellings in ihrem Verhältnis zur Religion, Memminger, Würzburg, 1926, p. 37.

75 BARTH, B.; Schellings Philosophie der Kunst, Gottliche Imagination und ästhetische Einbildungskraft, (Symposion Bd. 92), Alber, München 1991, p.53.

76 SW 390.

77 Cfr. Dietzsch, S., Natur-Kunst-Mythos. Beitrage zur Philosophie F. W. J. Schellings, Akademie Verlag, Berlin, 1978, pp. 29-33.

78 Knittermeyer, H., Schelling und die Romantische Schule, Reinhardt, München 1947, p. 343 . 
Bosch, M. (2016). «Significado estético del Sistema de Idealismo Trascendental», Contrastes. Revista Internacional de Filosofía, vol. XX, n², pp. 5-25.

Braun, O. (1906). Schellings geistige Wundlungen in den Jahren 1800-1810. Leipzig: Quelle und Meyer,.

De Torres, M. J. (1999). «Estética y religión en la filosofía del arte de Schelling», en Leyte, A. (Ed.) Una mirada a la filosofía de Schelling. Vigo: Universidad de Vigo.

Dietzsch, S. (1978). Natur-Kunst-Mythos. Beitrage zur Philosophie F. W. J. Schellings. Berlin: Akademie Verlag.

Finchman, R. M. (2015). «Reconciling Leibnizian monadology and kantian criticism», British Journal for the History of Philosophy, Vol. 23, No. 6, 1033-1055, DOI: 10.1080/09608788.2015.1105781.

Galán, I. (1999). El Romanticismo: F. W. J. Schelling o el arte divino. Madrid: Ediciones Endymion.

Genths, P. (1926). Die Identitäts Philosophie Schellings in ihrem Verhältnis zur Religion. Würzburg: Memminger.

Goudeli, K. (2012). «The potences of beauty: Schelling on the question of Nature and art», Comparative and Continental Philosophy, 4. 2, pp. 253-263.

Hartmann, N. (1974). Die Philosophie des Deutschen Idealismus. Berlin, New York: Walter de Gruyter.

Jaspers, K. (1986). Schelling, Grösse und Verhängnis. München: Piper.

Knittermeyer, H. (1947). Schelling und die Romantische Schule. München: Reinhardt.

Kogan, J. (1971). Arte y metafísica. Buenos Aires: Paidós.

Leyte, A. (2014). «Lo infinito romántico», Suplemento de Contrastes. Revista internacional de filosofía, 19, pp. 205-222.

Meier, F. (1961). Die Idee der Transzendentalphilosophie beim jungen Schelling. Winterthur: Keller.

Pareyson, L. (2003). Estetica dell'idealismo tedesco, (vol.III: Goethe e Schelling). Milano: Mursia.

Pereña, F. (2012). «Freiheit und System. Das Problem der Kompatibilität», Schellings Philosophie der Freiheit. Studien zu den Philosophischen Untersuchungen über das Wesen der menschlichen Freiheit. Ferrer und Pedro (Hrsg.). Würzburg: Ergon Verlag.

Perez-Borbujo, F. (2003). Schelling, el sistema de la libertad. Barcelona: Herder.

Rius, C. (2011). «La funció de l'artista en el pensament romàntic alemany», Comprendre, Vol. 13/2 Any, pp. 105-131.

Schelling, F. W. J. (1980). Philosophie der Kunst, Wissenschaftliche buch Gesellschaft Darmstadt, (Ausgabe Von 1859).

Schelling, F. W. J., Filosofía del arte, (Trad. V. López-Domínguez). Madrid: Tecnos.

Schlette, H. R. (1993). Weltseele. Geschichte und Hermeneutik. Frankfurt: Verlag J. Knecht.

Skarika, M. (1994). «Determinismo o libertad. La idea de libertad en Schelling», Anuario Filosófico, (27), pp. 719-730.

Stambovsky, P., "Idealism, scientia intuitiva, and scientific philosophy», The Review of Metaphysics 66 (December 2012): 339-352.

Thielke, P. (2013). «Recent work on early German Idealism 1781-1801», Journal of the History of Philosophy, vol. 51, no. 2, 149-192.

Warnek, P. (2004). «Schelling's Second Sailing», Epoché: A Journal for the History of Philosophy, vol.8, n.2, pp. 195-214.

Universitat Internacional de Catalunya

Magdalena Bosch

Facultad de Humanidades

C/ Immaculada, 22

08023 BarCELONA

mbosch@uic.es

[Artículo aprobado para publicación en enero de 2019] 